\title{
SURVEY ARTICLE: A TOUR OF THE WEAK AND STRONG LEFSCHETZ PROPERTIES
}

\author{
JUAN MIGLIORE AND UWE NAGEL
}

\section{CONTENTS}

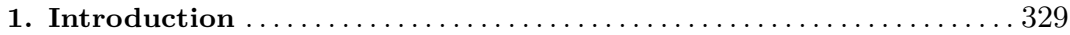

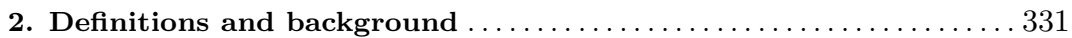

3. Complete intersections and Gorenstein algebras .............. 335

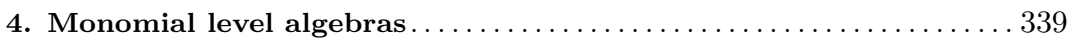

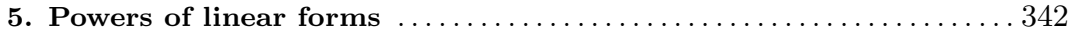

6. Connection between Fröberg's conjecture and the WLP ........ 347

7. Positive characteristics and enumerations $\ldots \ldots \ldots \ldots \ldots \ldots \ldots \ldots$

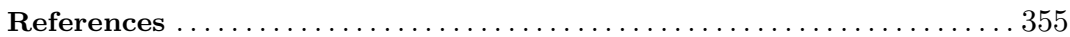

ABSTRACT. An artinian graded algebra, $A$, is said to have the weak Lefschetz property (WLP) if multiplication by a general linear form has maximal rank in every degree. A vast quantity of work has been done studying and applying this property, touching on numerous and diverse areas of algebraic geometry, commutative algebra and combinatorics. Amazingly, though, much of this work has a "common ancestor" in a theorem originally due to Stanley, although subsequently reproved by others. In this paper we describe the different directions in which research has moved starting with this theorem, and we discuss some of the open questions that continue to motivate current research.

1. Introduction. The weak and strong Lefschetz properties are strongly connected to many topics in algebraic geometry, commutative

This work was partially supported by two grants from the Simons Foundation (\#208579 to Juan Migliore and \#208869 to Uwe Nagel).

Received by the editors on September 26, 2011, and in revised form on December 16, 2012. 
algebra and combinatorics. Some of these connections are quite surprising and still not completely understood, and much work remains to be done. In this expository paper we give an overview of known results on the weak and strong Lefschetz properties, with an emphasis on the vast number of different approaches and tools that have been used, and connections that have been made with seemingly unrelated problems. One goal of this paper is to illustrate the variety of methods and connections that have been brought to bear on this problem for different families of algebras. We also discuss open problems.

Considering the amazing breadth and depth of results that have been found on this topic, and the tools and connections that have been associated with it, it is very interesting to note that, to a large degree, one result motivated this entire area. This result is the following. It was proved by Stanley [53] in 1980 using algebraic topology, by Watanabe in 1987 using representation theory, by Reid, Roberts and Roitman [48] in 1991 with algebraic methods, by Herzog and Popescu [30] (unpublished) in 2005, essentially with linear algebra, and it follows from the work of Ikeda [50] in 1996 using combinatorial methods.

Theorem 1.1. Let $R=k\left[x_{1}, \ldots, x_{r}\right]$, where $k$ has characteristic zero. Let $I$ be an artinian monomial complete intersection, i.e.,

$$
I=\left\langle x_{1}^{a_{1}}, \ldots, x_{r}^{a_{r}}\right\rangle .
$$

Let $\ell$ be a general linear form. Then, for any positive integers $d$ and $i$, the homomorphism induced by multiplication by $\ell^{d}$,

$$
\times \ell^{d}:[R / I]_{i} \rightarrow[R / I]_{i+d}
$$

has maximal rank. (In particular, this is true when $d=1$. )

This paper is organized around the ways that subsequent research owes its roots to this theorem.

Our account is by no means exhaustive. Fortunately, the manuscript [27] has appeared recently. It gives an overview of the Lefschetz properties from a different perspective, focusing more on the local case, representation theory and combinatorial connections different from those presented here. 
There is one topic that is neither treated in [27] nor here but that is worth mentioning briefly. In [39], examples of monomial ideals were exhibited that did not have the WLP but that could be deformed to ideals with the WLP. A systematic way for producing such deformations that preserve the Hilbert function has been proposed by Cook and Nagel in [19]. The idea is to lift the given monomial ideal to the homogenous ideal of a set of points and then pass to a general hyperplane section of the latter. It is shown in [19] that this procedure does indeed produce ideals with the WLP for a certain class of monomial ideals without the WLP.

In May 2011, the first author gave a talk at the Midwest Commutative Algebra and Geometry Conference at Purdue University on this topic. This paper is a vast expansion and extension of that talk, containing many more details and several new topics.

2. Definitions and background. Let $k$ be an infinite field. We will often take char $(k)=0$, but we will see that changing the characteristic produces interesting new questions (and even more interesting answers!).

Let $R=k\left[x_{1}, \ldots, x_{r}\right]$ be the graded polynomial ring in $r$ variables over $k$. Let

$$
A=R / I=\bigoplus_{i=0}^{n} A_{i}
$$

be a graded artinian algebra. Note that $A$ is finite dimensional over $k$.

Definition 2.1. For any standard graded algebra $A$ (not necessarily artinian), the Hilbert function of $A$ is the function

$$
\underline{h}_{A}: \mathbf{N} \longrightarrow \mathbf{N}
$$

defined by $\underline{h}_{A}(t)=\operatorname{dim}[A]_{t}$. One can express $\underline{h}_{A}$ as a sequence

$$
\left(h_{0}=1, h_{1}, h_{2}, h_{3}, \ldots\right) \text {. }
$$

An $O$-sequence is a sequence of positive integers that occurs as the Hilbert function of some graded algebra. When $A$ is Cohen-Macaulay, its $h$-vector is the Hilbert function of an artinian reduction of $A$. In 
particular, when $A$ is artinian, its Hilbert function is equal to its $h$ vector.

Definition 2.2. An almost complete intersection is a standard graded algebra $A=R / I$ which is Cohen-Macaulay, and for which the number of minimal generators of $I$ is one more than its codimension.

Definition 2.3. $A$ is level of Cohen-Macaulay type $t$ if its socle is concentrated in one degree (e.g., a complete intersection) and has dimension $t$.

Definition 2.4. Let $\ell$ be a general linear form. We say that $A$ has the weak Lefschetz property (WLP) if the homomorphism induced by multiplication by $\ell$,

$$
\times \ell: A_{i} \longrightarrow A_{i+1},
$$

has maximal rank for all $i$ (i.e., is injective or surjective). We say that $A$ has the strong Lefschetz property (SLP) if

$$
\times \ell^{d}: A_{i} \longrightarrow A_{i+d}
$$

has maximal rank for all $i$ and $d$ (i.e., is injective or surjective).

Remark 2.5. (a) One motivation for the work described in this paper is that something interesting should be going on if multiplication by a general linear form does not induce a homomorphism of maximal rank, even in one degree.

(b) Later we will see that there is a strong connection to Fröberg's conjecture. In this regard, we note that $\ell^{d}$ should not be considered to be a "general" form of degree $d$, since in the vector space $[R]_{d}(d>1)$, those forms that are pure powers of linear forms form a proper Zariskiclosed subset.

(c) Suppose that $\operatorname{deg} f=d$ and $\times f:[R / I]_{i} \rightarrow[R / I]_{i+d}$ has maximal rank, for all $i$. Pardue and Richert $[47]$ call such an $f$ semiregular. Reid, Roberts and Roitman [48] call such an $f$ faithful. If $\times f^{j}:[R / I]_{i} \rightarrow[R / I]_{i+d j}$ has maximal rank for all $i$ and all $j$, they call such an $f$ strongly faithful. So $R / I$ has the WLP if $R$ contains a linear 
faithful element, and $R / I$ has the SLP if $R$ contains a linear strongly faithful element.

(d) Several authors consider the question of the ranks that arise if $\times \ell^{d}$ is replaced by $\times F$ for a general $F$ of degree $d$. This is the essence of the Fröberg conjecture, is related to the WLP, and will be discussed below in Section 6 .

How do we determine if $R / I$ fails to have the WLP? Let $\ell$ be a general linear form and fix an integer $i$. Then we have an exact sequence

$$
[R / I]_{i-1} \stackrel{\times \ell}{\longrightarrow}[R / I]_{i} \longrightarrow[R /(I, \ell)]_{i} \longrightarrow 0
$$

Thus, $\times \ell$ fails to have maximal rank from degree $i-1$ to degree $i$ if and only if

$$
\operatorname{dim}[R /(I, \ell)]_{i}>\max \left\{0, \operatorname{dim}[R / I]_{i}-\operatorname{dim}[R / I]_{i-1}\right\} .
$$

More precisely, if we want to show that the WLP fails, it is enough to identify a degree $i$ for which we can produce one of the following two pieces of information:

(i) $\operatorname{dim}[R / I]_{i-1} \leq \operatorname{dim}[R / I]_{i}$ and $\operatorname{dim}[R /(I, \ell)]_{i}>\operatorname{dim}[R / I]_{i}-$ $\operatorname{dim}[R / I]_{i-1}$; in this case, we loosely say that WLP fails because of injectivity; or

(ii) $\operatorname{dim}[R / I]_{i-1} \geq \operatorname{dim}[R / I]_{i}$ and $\operatorname{dim}[R /(I, \ell)]_{i}>0$; in this case, we loosely say that WLP fails because of surjectivity.

In general, even identifying which $i$ is the correct place to look can be difficult. Then, determining which of (i) or (ii) holds, and establishing both inequalities, is often very challenging. This is where computer algebra programs have been very useful, in suggesting where to look and what to look for! On the other hand, to prove that $R / I$ does have the WLP, the following result is helpful:

Proposition 2.6 [39, Proposition 2.1]. Let $R / I$ be an artinian standard graded algebra, and let $\ell$ be a general linear form. Consider the homomorphisms $\phi_{d}:[R / I]_{d} \rightarrow[R / I]_{d+1}$ defined by multiplication by $\ell$, for $d \geq 0$.

(a) If $\phi_{d_{0}}$ is surjective for some $d_{0}$, then $\phi_{d}$ is surjective for all $d \geq d_{0}$. 
(b) If $R / I$ is level and $\phi_{d_{0}}$ is injective for some $d_{0} \geq 0$, then $\phi_{d}$ is injective for all $d \leq d_{0}$.

(c) In particular, if $R / I$ is level and $\operatorname{dim}[R / I]_{d_{0}}=\operatorname{dim}[R / I]_{d_{0}+1}$ for some $d_{0}$ then $R / I$ has the WLP if and only if $\phi_{d_{0}}$ is injective (and hence is an isomorphism).

This result helps to narrow down where one has to look, especially in the situation where we want to show that the WLP does hold. In this case you have to find the critical degrees and then show that surjectivity and (usually) injectivity do hold just in two (or occasionally one) spots.

In the case of one variable, the WLP and SLP are trivial since all ideals are principal. The case of two variables also has a nice result, at least in characteristic 0 :

Theorem $2.7[\mathbf{2 8}]$. If $\operatorname{char}(k)=0$ and $I$ is any homogeneous ideal in $k[x, y]$, then $R / I$ has the SLP.

The proof of this result used generic initial ideals with respect to the reverse lexicographic order. In the case of the WLP, it is not hard to show that the above theorem is true in any characteristic $([\mathbf{2 0}, \mathbf{3 5}$, 44]). However, the characteristic zero assumption cannot be omitted for guaranteeing the SLP. In fact, also the WLP may fail if there are at least three variables. The following is an easy exercise:

Lemma 2.8. Assume char $(k)=p$. Consider the ideal

$$
I=\left\langle x_{1}^{p}, \ldots, x_{r}^{p}\right\rangle \subset R=k\left[x_{1}, \ldots, x_{r}\right],
$$

where $r \geq 2$. Then

- $R /\left\langle x_{1}^{p}, \ldots, x_{r}^{p}\right\rangle$ fails the SLP for all $r \geq 2$.

- It fails the WLP for all $r \geq 3$.

- It has the WLP when $r=2$.

Remark 2.9. It was pointed out to us by the referee that, in order for failure of SLP to hold, one does not even need to take the exponents to be $p$ for all the variables. It suffices to take exponents at most 
$p$ summing to at least $p+r$. For example, $I=\left\langle x_{1}^{2+p-r}, x_{2}^{2}, \ldots, x_{r}^{2}\right\rangle$ works if $p \geq r$, and $I=\left\langle x_{1}^{2}, x_{2}^{2}, \ldots, x_{r}^{2}\right\rangle$ works if $p \leq r$. For the WLP one can as well use $I=\left\langle x_{1}^{p}, x_{2}^{p}, x_{3}^{2}, \ldots, x_{r}^{2}\right\rangle$.

In Section 7 we will discuss the presence of the WLP in positive characteristic in more detail.

A useful consequence of knowing that an algebra $A$ has the WLP or SLP is that its Hilbert function is unimodal. In fact, the Hilbert functions of algebras with the WLP have been completely classified:

Proposition 2.10 [28]. Let $\underline{h}=\left(1, h_{1}, h_{2}, \ldots, h_{s}\right)$ be a finite sequence of positive integers. Then $\underline{h}$ is the Hilbert function of a graded artinian algebra with the WLP if and only if the positive part of the first difference is an $O$-sequence and after that the first difference is non-positive until $\underline{h}$ reaches 0 . Furthermore, this is also a necessary and sufficient condition for $\underline{h}$ to be the Hilbert function of a graded artinian algebra with the SLP.

The challenge is thus to study the WLP and SLP (or their failures), and the behavior of the Hilbert function, for interesting families of algebras. Most of the results below fall into this description. It should also be noted that, conversely, some Hilbert functions $\underline{h}$ force any algebra with Hilbert function $\underline{h}$ to have the WLP; these were classified in $[44]$.

In the rest of this paper, we indicate different directions of research that have been motivated by Theorem 1.1; in most cases, there also remain many intriguing open problems.

3. Complete intersections and Gorenstein algebras. By semicontinuity, a consequence of Theorem 1.1 is that a general complete intersection with fixed generator degrees has the WLP and the SLP.

Question 3.1. Do all artinian complete intersections have the WLP or the SLP in characteristic 0 ?

We know that the answer is trivially "yes" in one and two variables. 
In three or more variables, the following is the most complete result known to date.

Theorem $3.2[\mathbf{2 8}]$. Let $R=k[x, y, z]$, where char $(k)=0$. Let $I=\left\langle F_{1}, F_{2}, F_{3}\right\rangle$ be a complete intersection. Then $R / I$ has the WLP.

The proof of this result introduced the use of the syzygy module of $I$, and its sheafification, the syzygy bundle. Subsequently, several papers have used the syzygy module to study the WLP for different kinds of ideals (see, e.g., $[\mathbf{1 1}, \mathbf{1 2}, \mathbf{2 0}, \mathbf{2 6}, \mathbf{3 9}, \mathbf{5 1}]$ ). In the case of complete intersections in $k[x, y, z]$, the syzygy bundle has rank 2. The WLP is almost immediate in the "easy" cases, and semi-stability and the Grauert-Mülich theorem give the needed information about $R /(I, \ell)$ in the "interesting" cases.

Remark 3.3. (i) The SLP is still wide open for complete intersections in three or more variables and, in fact, even the WLP is open for complete intersections of arbitrary codimension $\geq 4$. Some partial results on the WLP for arbitrary complete intersections in four variables have been obtained recently by the authors together with Boij and Miró-Roig, in work in progress.

(ii) It was conjectured by Reid, Roberts and Roitman [48] that the answer to both parts of Question 3.1 is yes.

We have seen that conjecturally (and known in special cases), all complete intersections have the WLP. Complete intersections are a special case of Gorenstein algebras. Does the conjecture extend to the Gorenstein case? That is,

Question 3.4. Do all graded artinian Gorenstein algebras have the WLP? If not, what are classes of artinian Gorenstein algebras that do have this property?

The answer to the first question is a resounding "no." Indeed, Stanley [52] in 1978 gave an example of an artinian Gorenstein algebra with Hilbert function $(1,13,12,13,1)$, which, because of non-unimodality, 
clearly does not have the WLP. Other examples of non-unimodality for Gorenstein algebras were given by Bernstein and Iarrobino [3], by Boij [4] and by Boij and Laksov [5]. Even among Gorenstein algebras with unimodal Hilbert functions, WLP does not necessarily hold. For instance, an example in codimension 4 was given by Ikeda [32] in 1996.

On the other hand, the problem in three variables is still wide open, with only special cases known (see for instance $[\mathbf{1}, \mathbf{4 5}]$ ):

Question 3.5. Does every artinian Gorenstein quotient of $k[x, y, z]$ have the WLP, provided char $(k)=0$ ? What about the SLP?

Given the complete intersection result for three variables mentioned above, this is a very natural and intriguing question.

In four variables, the result of Ikeda mentioned above shows that WLP need not hold. Nevertheless, the main result of [43] shows that, for small initial degree, the Hilbert functions are still precisely those of Gorenstein algebras with the WLP. More precisely, it was shown that, if the $h$-vector is $\left(1,4, h_{2}, h_{3}, h_{4}, \ldots\right)$ and $h_{4} \leq 33$, then this result holds. More recently, using the same methods, Seo and Srinivasan [51] extended this to $h_{4}=34$. Thus, the result holds for initial degree $\leq 4$.

Another interesting special case is the situation in which the generators of the ideal have small degree. We say that an algebra $R / I$ is presented by quadrics if the ideal $I$ is generated by quadrics. Such ideals occur naturally, for example, as homogeneous ideals of sufficiently positive embeddings of smooth projective varieties ([23]) or as StanleyReisner ideals of simplicial flag complexes ([55]). Gorenstein algebras presented by quadrics are studied, for example, in [42]. There, the following conjecture has been proposed.

Conjecture 3.6 [42]. Any artinian Gorenstein algebra presented by quadrics, over a field $k$ of characteristic zero, has the WLP.

The conjecture predicts, in particular, that if the socle degree is at least 3 then the multiplication by a general linear form from degree one to degree two is injective. Though this is established in some cases in [42], even this special case of the conjecture is open. 
The analog of Question 3.4 is also of interest for rings of positive dimension. If $A$ is a Gorenstein ring of dimension $d$, then $A$ is said to have the WLP if a general artinian reduction of $A$ has the WLP, that is, if $A /\left\langle L_{1}, \ldots, L_{d}\right\rangle$ has the WLP, where $L_{1}, \ldots, L_{d} \in A$ are general forms of degree 1. Recall that the Stanley-Reisner ring of the boundary complex of a convex polytope is a reduced Gorenstein ring. The socalled g-theorem classifies their Hilbert functions. The necessity of the conditions on the Hilbert function is a consequence of the following result by Stanley.

Theorem 3.7 [54]. The Stanley-Reisner ring of the boundary complex of a convex polytope over a field $k$ has the $\operatorname{SLP}$ if $\operatorname{char}(k)=0$.

The so-called g-conjecture states that the above-mentioned conditions on the Hilbert function characterize in fact the Hilbert functions of the Stanley-Reisner rings of triangulations of spheres. Note that there are many more such triangulations than boundary complexes of convex polytopes. In this regard, the following question merits highlighting:

Question 3.8. Does a general artinian reduction of a reduced, arithmetically Gorenstein set of points in $\mathbf{P}^{n}$ have the WLP, provided $\operatorname{char}(k)=0$ ?

We point out that, if this question has an affirmative answer, then, by the main result of $[\mathbf{4 1}]$, we have a classification of the Hilbert functions of reduced, arithmetically Gorenstein schemes: their $h$-vectors are precisely the SI-sequences, meaning that they are symmetric, with the first half itself a differentiable $O$-sequence.

An affirmative answer to Question 3.8 would also imply the gconjecture, thus providing a characterization of the face vectors of triangulations of a sphere. Moreover, the methods used to establish the WLP could lead to information about the face vectors of triangulations of other manifolds as well. In fact, Novik and Swartz [46, Theorem 1.4], show that a certain quotient of the Stanley-Reisner ring of any orientable $k$-homology manifold without boundary is a Gorenstein ring. Kalai conjectured that this Gorenstein ring has the SLP. If true, this would establish new restrictions on the face vectors of these complexes. 
A special case of Kalai's conjecture has been proved in Theorem 1.6 of [46].

4. Monomial level algebras. Note that $R /\left\langle x_{1}^{a_{1}}, \ldots, x_{r}^{a_{r}}\right\rangle$ is also a level artinian monomial algebra.

Question 4.1. Which (if any) level artinian monomial algebras fail the WLP or SLP?

The first result in this direction is a positive one:

Theorem 4.2 (Hausel [29, Theorem 6.2]). Let $A$ be a monomial artinian level algebra of socle degree $e$. If the field $k$ has characteristic zero, then for a general linear form $\ell$, the induced multiplication

$$
\times \ell: A_{j} \longrightarrow A_{j+1}
$$

is an injection, for all $j=0,1, \ldots,\lfloor(e-1) / 2\rfloor$. In particular, over any field the sequence

$$
1, h_{1}-1, h_{2}-h_{1}, \ldots, h_{\lfloor(e-1) / 2\rfloor+1}-h_{\lfloor(e-1) / 2\rfloor}
$$

is an O-sequence, i.e., the "first half" of $\underline{h}$ is a differentiable $O$ sequence.

Thus, roughly "half" the algebra does satisfy the WLP. What about the second half? The first counterexample was due to Zanello ([57, Example 7]), who showed that the WLP does not necessarily hold for monomial level algebras even in three variables. His example had $h$ vector $(1,3,5,5)$. Subsequently, Brenner and Kaid ([11, Example 3.1]) produced an example of a level artinian monomial almost complete intersection algebra that fails the WLP; this algebra has $h$-vector $(1,3,6,6,3)$ and, in particular, Cohen-Macaulay type 3 . The study of such almost complete intersections was continued by Migliore, MiróRoig and Nagel [39], and more recently by Cook and Nagel [18, 20] (see also Section 7).

The Hilbert functions of the algebras considered in Question 4.1 are of great interest in a number of areas. In fact, they are better known under a different name. 
Definition 4.3. A pure $O$-sequence of type $t$ in $r$ variables is the Hilbert function of a level artinian monomial algebra $k\left[x_{1}, \ldots, x_{r}\right] / I$ of Cohen-Macaulay type $t$.

Question 4.4. We have already seen that level artinian monomial algebras do not necessarily have the WLP. Nevertheless, are their Hilbert functions unimodal? That is, are all pure O-sequences unimodal? If not, can we find subfamilies, depending upon the type $t$ and/or the number of variables $r$, that are unimodal? And, if they are not necessarily unimodal, "how non-unimodal" can they be?

Remark 4.5. If $I$ is a monomial ideal in $R=k\left[x_{1}, \ldots, x_{r}\right]$, then the linear form $\ell=x_{1}+\cdots+x_{r}$ is "general enough" to determine whether $R / I$ has the WLP or SLP. This observation has been extremely useful in simplifying calculations to show the existence or failure of the WLP. In [39, Proposition 2.2], this was stated for the WLP, but the same proof also gives it for the SLP.

For the remainder of this section we will assume that $k$ has characteristic 0 , unless explicitly mentioned otherwise. We have seen that, in one or two variables, we always have the WLP (and even SLP). Turning to the next case, the following seemingly simple result in fact has a very intricate and long proof. It illustrates the subtlety of these problems.

Theorem 4.6 [8, Theorem 6.2]. A level artinian monomial algebra of type 2 in three variables has the WLP. ${ }^{1}$

Of course, this has the following consequence.

Corollary 4.7. A pure O-sequence of type 2 in three variables is unimodal.

The monograph [6] gave a careful study of families of level artinian monomial algebras that fail the WLP. As a consequence, we have the following conclusion. 
Theorem 4.8 [6]. If $R=k\left[x_{1}, \ldots, x_{r}\right]$ and $R / I$ is a level artinian monomial algebra of type $t$, then, for all $r$ and $t$, examples exist where the WLP fails, except if:

- $r=1$ or 2 ;

- $t=1$ (this is Theorem 1.1);

- $r=3, t=2$ (this is Theorem 4.6).

In particular, the first case where WLP can fail is when $r=3$ and $t=3$. This occurs, for instance, if $I=\left\langle x^{3}, y^{3}, z^{3}, x y z\right\rangle$ (see $[\mathbf{1 1}$, Example 3.1]). Nevertheless, Boyle has shown that, despite the failure of the WLP, all level artinian monomial algebras with $r=3$ and $t=3$ have strictly unimodal Hilbert function (that is, in addition to being unimodal, once the function decreases then it is strictly decreasing from that point until it reaches zero):

Theorem 4.9 [9]. Any pure $O$-sequence of type 3 in three variables is strictly unimodal.

In more variables, the first case where the WLP can fail is when $r=4$ and $t=2$. Here again, Boyle has shown that, nevertheless, such algebras have strictly unimodal Hilbert function:

Theorem 4.10 [10]. Any pure $O$-sequence of type 2 in four variables is strictly unimodal.

Since the WLP is not available in these cases, Boyle's method is a classification theorem followed by a decomposition of the ideals and a careful analysis of sums of Hilbert functions of complete intersections.

However, there is no hope of such a result for all pure $O$-sequences, even when $r=3$ :

Theorem 4.11 [8]. Let $M$ be any positive integer, and fix an integer $r \geq 3$. Then there exists a pure O-sequence in $r$ variables which is non-unimodal, having exactly $M$ maxima. 
In view of the last two results, we have the following natural question.

Question 4.12. What is the smallest socle degree and (separately) the smallest socle type $t$ for which non-unimodal pure O-sequences exist? This is especially of interest when $r=3$.

In [6], Boij and Zanello produced a non-unimodal example with $r=3$ and socle degree 12. In [8], for $r=3$, we produced a non-unimodal example for socle type $t=14$. It was also shown that pure $O$-sequences can fail unimodality if and only if the socle degree is at least 4 (but one may need many variables for small socle degree).

It is also natural to ask how things change when you remove "monomial" and ask about artinian level algebras. Some work in progress by Boij, Migliore, Miró-Roig, Nagel and Zanello indicates that the behavior of such algebras from the point of view of the Hilbert function can become surprisingly worse, in the sense that dramatic non-unimodality is possible even in early degrees, which would violate Hausel's theorem (Theorem 4.2) for instance, in the monomial case.

5. Powers of linear forms. In this section we always assume that $k$ has characteristic zero. Note that $x_{i}$ is a linear form, and that if $L_{1}, \ldots, L_{n}(n \geq r)$ are general linear forms, then, without loss of generality, (by a change of variables) we can assume that $L_{1}=x_{1}, \ldots, L_{r}=x_{r}$. Thus, Theorem 1.1 is also a result about ideals generated by powers of linear forms. It says that, in $k\left[x_{1}, \ldots, x_{r}\right]$, an ideal generated by powers of $r$ general linear forms has the WLP and the SLP. It also leads to an interesting connection to Fröberg's conjecture, which we discuss in Section 6 .

Question 5.1. Which ideals generated by powers of general linear forms define algebras that fail the WLP or SLP?

We saw in Theorem 2.7 that all such ideals (and in fact all homogeneous ideals) in two variables satisfy both the WLP and the SLP. More surprisingly, Schenck and Seceleanu showed a similar result in three variables: 
Theorem $5.2[51]$. Let $R=k[x, y, z]$, where $\operatorname{char}(k)=0$. Let $I=\left\langle L_{1}^{a_{1}}, \ldots, L_{m}^{a_{m}}\right\rangle$ be any ideal generated by powers of linear forms. Then $R / I$ has the WLP.

A shorter proof of this result is given in [40]. One reason that it is surprising is that the same is not true for SLP. For instance, if $I=\left\langle L_{1}^{3}, L_{2}^{3}, L_{3}^{3}, L_{4}^{3}\right\rangle$ (where $L_{i}$ is general in $k[x, y, z]$ ), then $\left(\times \ell^{3}\right)$ fails to have maximal rank. The case of three variables acts as a bridge case: we will see that, for four or more variables, even WLP fails very commonly. Some recent work in this area was motivated by the following example of Migliore, Miró-Roig and Nagel:

Example 5.3 [39]. Let $r=4$. Consider the ideal $I=\left\langle x_{1}^{N}, x_{2}^{N}, x_{3}^{N}\right.$, $\left.x_{4}^{N}, L^{N}\right\rangle$ for a general linear form $L$. By computation using CoCoA, $R / I$ fails the WLP, for $N=3, \ldots, 12$.

There are some natural questions arising from this example:

Problem 5.4. $\quad$ - Prove the failure of the WLP in Example 5.3 for all $N \geq 3$.

- What happens for mixed powers?

- What happens for almost complete intersections, that is, for $r+1$ powers of general linear forms in $r$ variables when $r>4$ ?

- What about more than $r+1$ powers of general linear forms?

This example motivated two different projects at the same time: by Migliore, Miró-Roig, Nagel [40] and by Harbourne, Schenck and Seceleanu [26]. Both of these papers used the dictionary between ideals of powers of general linear forms and ideals of fat points in projective space, provided by the following important result of Emsalem and Iarrobino:

Theorem $5.5[24]$. Let

$$
\left\langle L_{1}^{a_{1}}, \ldots, L_{n}^{a_{n}}\right\rangle \subset k\left[x_{1}, \ldots, x_{r}\right]
$$

be an ideal generated by powers of $n$ linear forms. Let $\wp_{1}, \ldots, \wp_{n}$ be 
the ideals of the $n$ points in $\mathbf{P}^{r-1}$ corresponding to the linear forms. Then, for any integer $j \geq \max \left\{a_{i}\right\}$,

$$
\operatorname{dim}_{k}\left[R /\left\langle L_{1}^{a_{1}}, \ldots, L_{n}^{a_{n}}\right\rangle\right]_{j}=\operatorname{dim}_{k}\left[\wp_{1}^{j-a_{1}+1} \cap \cdots \cap \wp_{n}^{j-a_{n}+1}\right]_{j} .
$$

One important difference between the two papers is that [26] assumed that the powers are uniform, and usually that the powers are "large enough." Usually they allow more than $r+1$ forms. On the other hand, most of the results in [40] allow mixed powers. We quote some of the results of these two papers. Together they form a nice start to an interesting topic. The conjectures listed later indicate that more work is to be done!

Theorem $5.6[26]$. Let

$$
I=\left\langle L_{1}^{t}, \ldots, L_{n}^{t}\right\rangle \subset k\left[x_{1}, x_{2}, x_{3}, x_{4}\right],
$$

with $L_{i} \in R_{1}$ generic. If $n \in\{5,6,7,8\}$, then the WLP fails, respectively, for $t \geq\{3,27,140,704\}$.

Theorem $5.7[\mathbf{2 6}]$. For

$$
I=\left\langle L_{1}^{t}, \ldots, L_{2 k+1}^{t}\right\rangle \subset R=k\left[x_{1}, \ldots, x_{2 k}\right]
$$

with $L_{i}$ generic linear forms, $k \geq 2$ and $t \gg 0, R / I$ fails the WLP.

(See also Theorem 5.10 below.) The following result gives the most complete picture to date, about the case of four variables, when the exponents are not assumed to be uniform and the ideal is assumed to be an almost complete intersection (i.e. the number of minimal generators is one more than the number of variables). It summarizes several theorems in [40, Section 3], and we refer to that paper for the more detailed individual statements.

Theorem 5.8 (Four variables, [40]). Let

$$
I=\left\langle L_{1}^{a_{1}}, L_{2}^{a_{2}}, L_{3}^{a_{3}}, L_{4}^{a_{4}}, L_{5}^{a_{5}}\right\rangle \subset R=k\left[x_{1}, x_{2}, x_{3}, x_{4}\right],
$$


where all $L_{i}$ are generic. Without loss of generality, assume that $a_{1} \leq a_{2} \leq a_{3} \leq a_{4} \leq a_{5}$. Set

$$
\lambda= \begin{cases}\left(a_{1}+a_{2}+a_{3}+a_{4}\right) / 2-2 & \text { if } a_{1}+a_{2}+a_{3}+a_{4} \text { is even } \\ \left(a_{1}+a_{2}+a_{3}+a_{4}-7\right) / 2 & \text { if } a_{1}+a_{2}+a_{3}+a_{4} \text { is odd. }\end{cases}
$$

(a) If $a_{5} \geq \lambda$, then $R / I$ has the WLP.

(b) If $a_{1}=2$, then $R / I$ has the WLP.

(c) Most other cases (explicitly described in terms of $a_{1}, a_{2}, a_{3}, a_{4}$ ) are proven to fail the WLP.

(d) For the few open cases, experimentally sometimes the WLP holds and sometimes it does not.

Notice that the case where all the $a_{i}$ are equal and at least 3 is contained in Theorem 5.6.

In more than four variables, it becomes progressively more difficult to obtain results for mixed powers. We have the following partial result.

Theorem 5.9 (Five variables, almost uniform powers [40]). Assume $r=5$. Let $L_{1}, \ldots, L_{6}$ be general linear forms. Let $e \geq 0$ and

$$
I=\left\langle L_{1}^{d}, L_{2}^{d}, L_{3}^{d}, L_{4}^{d}, L_{5}^{d}, L_{6}^{d+e}\right\rangle .
$$

(a) If $e=0$, then $R / I$ fails the WLP if and only if $d>3$.

(b) If $e \geq 1$ and $d$ is odd, then $R / I$ has the WLP if and only if $e \geq(3 d-5) / 2$.

(c) If $e \geq 1$ and $d$ is even, then $R / I$ has the WLP if and only if $e \geq(3 d-8) / 2$.

We also have the following improvement of Theorem 5.7, which has the additional assumption that $t \gg 0$.

Theorem 5.10 (Even number of variables, uniform powers $[40]$ ). Let

$$
I=\left\langle L_{1}^{t}, \ldots, L_{2 k+1}^{t}\right\rangle \subset R=k\left[x_{1}, \ldots, x_{2 k}\right]
$$


with $L_{i}$ generic linear forms and $k \geq 2$. Then $R / I$ fails the WLP if and only if $t>1$.

(The case $k=2$ is contained in Theorem 5.8.)

What about an odd number of variables? Here is a result for seven variables:

Theorem $5.11[40]$. Let

$$
I=\left\langle L_{1}^{t}, \ldots, L_{8}^{t}\right\rangle \subset k\left[x_{1}, \ldots, x_{7}\right]
$$

where $L_{1}, \ldots, L_{8}$ are general linear forms.

- If $t=2$, then $R / I$ has the WLP.

- If $t \geq 4$, then $R / I$ fails the WLP.

Interestingly, for $t=3$, CoCoA [16] says that the WLP fails, but we do not have a proof. We can believe a computer that says that the WLP holds, but otherwise we have to be skeptical about whether its choice of forms was "general enough."

For these results, sometimes it was necessary to prove failure of surjectivity (when $h_{i-1} \geq h_{i}$ in the relevant degrees), sometimes failure of injectivity (when $h_{i-1} \leq h_{i}$ ), and sometimes we had to show that the WLP does hold. These present quite different challenges.

After making the translation to fat points, as described above, the first difficulty is to determine the degrees where WLP fails. Then, it is necessary to find the dimension of a linear system of surfaces in a suitable projective space vanishing to prescribed multiplicity at a general set of suitably many points. To do this, in [40], Cremona transformations and works of Dumnicki (2009), Laface-Ugaglia (2006) and De Volder-Laface (2007) were used as the main tools, plus ad hoc methods. These Cremona transformation results are central to the proofs in $[\mathbf{4 0}]$.

Much remains to be shown on this topic. Here are two conjectures from $[\mathbf{2 6}, \mathbf{4 0}]$. 
Conjecture $5.12[26]$. For $I=\left\langle L_{1}^{t}, \ldots, L_{n}^{t}\right\rangle \subset R=\left[x_{1}, \ldots, x_{r}\right]$ with $L_{i} \in R_{1}$ generic and $n \geq r+1 \geq 5$, the WLP fails for all $t \gg 0$.

Conjecture 5.13 [40]. Let $R=k\left[x_{1}, \ldots, x_{2 n+1}\right]$. Let $L_{1}, \ldots, L_{2 n+2}$ be general linear forms and $I=\left\langle L_{1}^{d}, \ldots, L_{2 n+1}^{d}, L_{2 n+2}^{d}\right\rangle$.

- If $n=3$ and $d=3$, then $R / I$ fails the WLP. (This is the only open case in Theorem 5.11.)

- If $n \geq 4$, then $R / I$ fails the WLP if and only if $d>1$.

These conjectures are supported by a great deal of computer evidence, using CoCoA [16] and Macaulay2 [25].

6. Connection between Fröberg's conjecture and the WLP. In this section we continue to assume that our field has characteristic zero. Closely related to the SLP is the so-called maximal rank property (MRP), which just replaces $\ell^{d}$ by a general form of degree $d$ in Definition 2.4. Nevertheless, it is known that the MRP does not imply the SLP. See $[\mathbf{3 8}, \mathbf{5 8}]$ for some connections between these two properties.

One way of stating Fröberg's conjecture is as follows.

Conjecture 6.1 (Fröberg). Any ideal of general forms has the MRP. More precisely, fix positive integers $a_{1}, \ldots, a_{s}$ for some $s>1$. Let $F_{1}, \ldots, F_{s} \subset R=k\left[x_{1}, \ldots, x_{r}\right]$ be general forms of degrees $a_{1}, \ldots, a_{s}$, respectively, and let $I=\left\langle F_{1}, \ldots, F_{s}\right\rangle$. Then, for each $i, 2 \leq i \leq s$, and for all $t$, the multiplication by $F_{i}$ on $R /\left\langle F_{1}, \ldots, F_{i-1}\right\rangle$ has maximal rank, from degree $t-a_{i}$ to degree $t$. As a result, the Hilbert function of $R / I$ can be computed inductively.

This conjecture is known to be true in two variables. This follows, for example, from Theorem 2.7. In three variables it was shown to be true by Anick [2]. In this section, we explore the following natural questions.

Question 6.2. What is the Hilbert function of an ideal generated by powers of general linear forms of degrees $a_{1}, \ldots, a_{n}$ ? In particular, is 
it the same as the Hilbert function predicted by Fröberg? What, if any, is the connection to the WLP?

Theorem 1.1 says that, when $n=r+1$, the answer to the second question is yes.

The fact that the answer is often "no" for $n=r+2$ was first observed by Iarrobino $[\mathbf{3 1}]$. Chandler $[\mathbf{1 3}, \mathbf{1 4}]$ also gave some results in this direction. Concerning the connection to the WLP, the following result of Migliore, Miró-Roig and Nagel gives a partial answer.

Proposition 6.3 [40]. (a) If Fröberg's conjecture is true for all ideals generated by general forms in $r$ variables, then all ideals generated by general forms in $r+1$ variables have the WLP.

(b) Let $R=k\left[x_{1}, \ldots, x_{r+1}\right]$, let $\ell \in R$ be a general linear form and let $S=R /\langle\ell\rangle \cong k\left[x_{1}, \ldots, x_{r}\right]$. Fix positive integers $s, d_{1}, \ldots, d_{s}, d_{s+1}$. Let $L_{1}, L_{2}, \ldots, L_{s}, L_{s+1} \in R$ be linear forms. Denote by - the restriction from $R$ to $S \cong R /\langle\ell\rangle$. Make the following assumptions:

(i) The ideal $I=\left\langle L_{1}^{d_{1}}, \ldots, L_{s}^{d_{s}}\right\rangle$ has the WLP.

(ii) The multiplication $\times \bar{L}_{s+1}^{d_{s+1}}:[S / \bar{I}]_{j-d_{s+1}} \rightarrow[S / \bar{I}]_{j}$ has maximal rank.

Then $R /\left\langle L_{1}^{d_{1}}, \ldots, L_{s+1}^{d_{s+1}}\right\rangle$ has the WLP.

Remark 6.4. (a) Part of this result was in fact contained in the paper [38] of Migliore and Miró-Roig. It was used there to show that any ideal of general forms in $k\left[x_{1}, x_{2}, x_{3}, x_{4}\right]$ satisfies the WLP, because Anick [2] had shown much earlier that any ideal of general forms in $k\left[x_{1}, x_{2}, x_{3}\right]$ satisfies Fröberg's conjecture.

(b) It was shown in [40] that this result also leads to a short proof of Theorem 5.2. The point is that the restriction of such ideals corresponds to an ideal in $k[x, y]$, and in characteristic zero all such ideals have the SLP by Theorem 2.7.

The following corollary was also shown in [40]:

Corollary 6.5 [40]. Assume the characteristic is zero. Let $R=$ $k\left[x_{1}, \ldots, x_{r+1}\right]$, let $\ell \in R$ be a general linear form and let $S=$ $R /\langle\ell\rangle \cong k\left[x_{1}, \ldots, x_{r}\right]$. For integers $d_{1}, \ldots, d_{r+2}$, if an ideal of the 
form $\left\langle L_{1}^{d_{1}}, \ldots, L_{r+2}^{d_{r+2}}\right\rangle \subset R$ of powers of general linear forms fails to have the WLP, then an ideal of powers of general linear forms $\left\langle\bar{L}_{1}^{d_{1}}, \ldots, \bar{L}_{r+2}^{d_{r+2}}\right\rangle \subset S$ fails to have the Hilbert function predicted by Fröberg's conjecture.

Thus, the results in the previous section give additional insight to the observations of Iarrobino $[\mathbf{3 1}]$ and Chandler $[\mathbf{1 3}, \mathbf{1 4}]$ that, when $n=r+2$, there are many cases when an ideal of powers of general linear forms does not have the same Hilbert function as that predicted by Fröberg for general forms. Since Theorem 5.8 covers almost all possible choices of exponents, it gives a much more complete answer to the question of exactly which powers of five general linear forms in three variables fail to have the Fröberg-predicted Hilbert function, contrasting with the result of Anick which says that an ideal of general forms of any fixed degrees in three variables does have the predicted Hilbert function. Theorems 5.9 and 5.10 provide new partial answers (via Corollary 6.5) in the case of more variables.

Example 6.6. Let $R=k\left[x_{1}, x_{2}, x_{3}, x_{4}\right]$. Let $L_{1}, L_{2}, L_{3}, L_{4}, L_{5}$ and $\ell$ be general linear forms. Let $S=R /\langle L\rangle \cong k[x, y, z]$. Let $I=\left\langle L_{1}^{3}, L_{2}^{3}, L_{3}^{3}, L_{4}^{3}, L_{5}^{3}\right\rangle$ (the smallest case in Example 5.3 above). The Hilbert function of $R / I$ is $(1,4,10,15,15,6)$. We have

$$
[R / I]_{3} \stackrel{\times \ell}{\longrightarrow}[R / I]_{4} \longrightarrow[R /(I, \ell)]_{4} \longrightarrow 0 .
$$

We saw that WLP fails, and in fact

$$
\operatorname{dim}[R /(I, \ell)]_{4}=1 .
$$

Notice that $R /(I, \ell) \cong S / J$, where $J$ is the ideal of cubes of five general linear forms in $k[x, y, z]$. Thus, $\operatorname{dim}[S / J]_{4}=1$.

On the other hand, let $K$ be the ideal of five general cubics in $S$. Fröberg predicts (and Anick proves) that $\operatorname{dim}[S / K]_{4}=0$. Thus, $J$ does not have the Hilbert function predicted by Fröberg.

In fact, whenever we prove that an ideal of $n$ powers of general linear forms fails the WLP (for specified exponents), then for some subset of these powers of general linear forms, the same number and powers 
of general linear forms in one fewer variable fails to have Fröberg's predicted Hilbert function.

7. Positive characteristics and enumerations. Considering Theorem 1.1 again, we saw in Lemma 2.8 that the assumption on the characteristic of the base field cannot be omitted.

Question 7.1. What happens in Theorem 1.1 if we allow the characteristic to be positive?

Actually, investigating the dependence of the WLP on the characteristic makes sense whenever the ideal can be defined over the integers. This applies to all monomial ideals. In fact, in this case one has the following result.

Proposition 7.2 [20, Lemma 2.6]. Let $I \subset R$ be a monomial ideal. If $R / I$ has the WLP when char $(k)=0$, then $R / I$ has the WLP whenever char $(k)$ is sufficiently large.

The proof is based on two observations that have their origin in [39]. For a monomial ideal, one can check the WLP by considering the specific linear form $\ell=x_{1}+\cdots+x_{r}$. Thus, the maximal rank property of the multiplications by $\ell$ is governed by integer matrices. Their determinants have only finitely many prime divisors if they do not vanish.

It also follows that $R / I$ fails the WLP in every positive characteristic if it fails the WLP in characteristic zero.

Proposition 7.2 motivates the following problem.

Question 7.3. Let $I \subset R$ be a monomial ideal such that $R / I$ has the WLP when char $(k)=0$. What are the (finitely many) field characteristics such that $R / I$ fails the WLP?

This turns out to be a rather subtle problem. It was first considered in $[39]$ in the case of a certain almost complete intersection in three variables. Recall that a monomial almost complete intersection in three 
variables is an ideal of the form

$$
I=I_{a, b, c, \alpha, \beta, \gamma}=\left\langle x^{a}, y^{b}, z^{c}, x^{\alpha} y^{\beta} z^{\gamma}\right\rangle
$$

If the syzygy bundle of $I$ is not semi-stable or its first Chern class is not divisible by three, then $R / I$ has the WLP in characteristic zero (see $[\mathbf{1 1}, \mathbf{3 9}]$ ). However, if the syzygy bundle satisfies both conditions, then deciding the WLP is more difficult and very subtle on the one side. On the other side, the investigations in this case have brought to light surprising connections to combinatorial problems.

In fact, if the syzygy bundle of $I$ is semi-stable and its first Chern class is divisible by three, then $R / I$ has the WLP if and only if the multiplication by $\ell$ in a certain degree is an isomorphism or, equivalently, a certain integer square matrix has a non-vanishing determinant. This has first been observed in the special case, where $R / I$ is level, in [39] and then for arbitrary almost complete intersections in [20]. The first connection to combinatorics was made by Cook and Nagel in $[\mathbf{1 8}$, Section 4]. There it was observed that the determinant deciding the WLP for certain families of monomial almost complete intersections is the number of lozenge tilings of some hexagon, which is given by a formula of MacMahon. Lozenge tilings of a hexagon are in bijection to other well-studied combinatorial objects such as, for example, plane partitions and families of non-intersecting lattice paths.

Independently of $[\mathbf{1 8}]$, but subsequent to it, Li and Zanello studied the WLP in the case of the complete intersections $R /\left\langle x^{a}, y^{b}, z^{c}\right\rangle$ in [35], and they also related MacMahon's numbers of plane partitions to the failure of the WLP:

Theorem 7.4 [35]. For any given positive integers $a, b, c$, the number of plane partitions contained inside an $a \times b \times c$ box is divisible by $a$ prime $p$ if and only if the algebra $k[x, y, z] /\left\langle x^{a+b}, y^{a+c}, z^{b+c}\right\rangle$ fails to have the WLP when char $(k)=p$.

(This connection is already implicitly contained in [18], although it was only made explicit in the proof of Corollary 6.5 in [20].) Next, Chen, Guo, Jin and Liu [15], explained bijectively the result by Li and Zanello for complete intersections. Both [18, 35] have been substantially extended in [20]. Here the bijective approach of $[\mathbf{1 5}]$ 
was extended to almost complete intersections, and further relations between the presence of the WLP and difficult counting problems in combinatorics have been given. In the remainder of this section, we give an overview of some of the results of $[\mathbf{2 0}]$ which illustrate this fascinating connection.

We focus on the most difficult case, in which the presence of the WLP is a priori not even known in characteristic zero, that is, we assume that the syzygy bundle of the almost complete intersection $I=I_{a, b, c, \alpha, \beta, \gamma}=\left\langle x^{a}, y^{b}, z^{c}, x^{\alpha} y^{\beta} z^{\gamma}\right\rangle$ is semi-stable in characteristic zero and its first Chern class is divisible by three. By [20, Proposition 3.3 , this is exactly true if and only if the following conditions are all satisfied:

(i) $s:=(a+b+c+\alpha+\beta+\gamma) / 3-2$ is an integer,

(ii) $0 \leq M$,

(iii) $0 \leq A \leq \beta+\gamma$,

(iv) $0 \leq B \leq \alpha+\gamma$, and

(v) $0 \leq C \leq \alpha+\beta$,

where

$$
\begin{aligned}
A & :=s+2-a, \\
B & :=s+2-b, \\
C & :=s+2-c, \text { and } \\
M & :=s+2-(\alpha+\beta+\gamma) .
\end{aligned}
$$

The above conditions have a geometric meaning. In fact, due to Theorem 4.1 in [20], they guarantee that $I$ can be related to a hexagonal region with a hole, which is called the punctured hexagon $H=H_{a, b, c, \alpha, \beta, \gamma}$ associated to $I=I_{a, b, c, \alpha, \beta, \gamma}$ (see Figure 1).

There are two square matrices that govern the WLP of the ideal $I$. In fact, $I$ has the WLP if and only if the multiplication $[R / I]_{s} \stackrel{x \ell}{\rightarrow}$ $[R / I]_{s+1}$ is bijective or, equivalently, $[R /(I, \ell)]_{s+1}=0$. The latter condition means that a certain $(C+M) \times(C+M)$ matrix, $N=$ $N_{a, b, c, \alpha, \beta, \gamma}$, with binomial coefficients as entries is regular. The above multiplication map can be described by a much larger zero-one square matrix, $Z=Z_{a, b, c, \alpha, \beta, \gamma}$. The above-mentioned equivalence implies that the determinants of $N$ and $Z$ have the same prime divisors. However, 


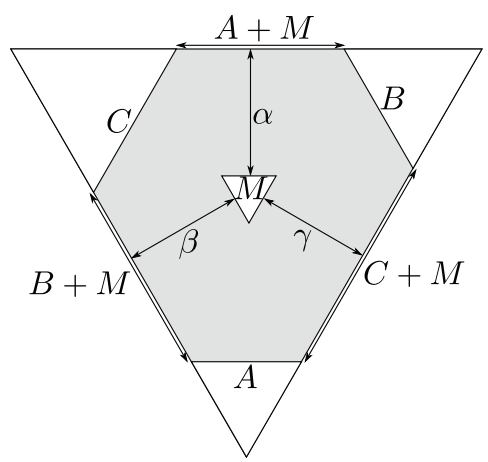

FIGURE 1. Punctured hexagon $H_{a, b, c, \alpha, \beta, \gamma}$ (shadowed) associated to $I_{a, b, c, \alpha, \beta, \gamma}$.

much more is true. Both determinants have the same absolute value, which has combinatorial interpretations.

Theorem 7.5 [20, Theorems 4.4, 4.6 and 5.4]. Adopt the above assumptions. Then the following conditions are equivalent:

(a) $I_{a, b, c, \alpha, \beta, \gamma}$ has the WLP if the characteristic of the base field $k$ is $p \geq 0$.

(b) $p$ does not divide the enumeration $\left|\operatorname{det} N_{a, b, c, \alpha, \beta, \gamma}\right|$ of signed lozenge tilings of the associated punctured hexagon $H_{a, b, c, \alpha, \beta, \gamma}$.

(c) $p$ does not divide the enumeration $\left|\operatorname{det} Z_{a, b, c, \alpha, \beta, \gamma}\right|$ of signed perfect matchings of the bipartite graph associated to $H_{a, b, c, \alpha, \beta, \gamma}$.

In particular, $\left|\operatorname{det} N_{a, b, c, \alpha, \beta, \gamma}\right|=\left|\operatorname{det} Z_{a, b, c, \alpha, \beta, \gamma}\right|$.

A lozenge is a rhombus with unit side-lengths and angles of $60^{\circ}$ and $120^{\circ}$. Lozenges have also been called calissons and diamonds in the literature. A perfect matching of a graph is a set of pairwise nonadjacent edges such that each vertex of the graph is matched. We refer to [20] for more details, in particular for assigning the signs, although Figure 2 indicates an associated lozenge tiling and a perfect matching.

Theorem 7.5 has been used to establish the WLP of $I_{a, b, c, \alpha, \beta, \gamma}$ in many new cases. The results also lend further evidence to a conjectured characterization of the presence of the WLP of $I_{a, b, c, \alpha, \beta, \gamma}$ in case $I_{a, b, c, \alpha, \beta, \gamma}$ is level that has been proposed in $[\mathbf{3 9}]$. 


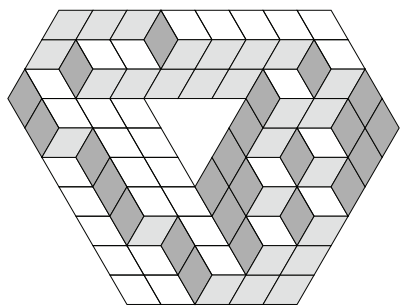

Hexagon tiling by lozenges

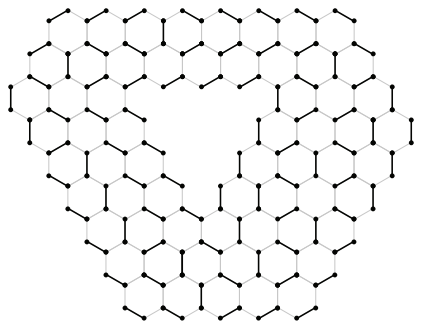

Perfect matching of edges

FIGURE 2. A lozenge tiling and its associated perfect matching.

The determinants occurring in Theorem 7.5 can be rather big.

Example 7.6. Consider the ideal

$$
I=\left\langle x^{14}, y^{21}, z^{25}, x^{2} y^{9} z^{13}\right\rangle
$$

Then the absolute value of the corresponding determinants is (see $[\mathbf{1 8}$, Remark 4.8])

$$
2 \cdot 3^{2} \cdot 5^{3} \cdot 11^{4} \cdot 13^{5} \cdot 19 \cdot 23^{3} \cdot 29 \cdot 5011
$$

Hence, $R / I$ fails the WLP if and only if the characteristic of $k$ is any of the nine listed prime divisors.

In the situation of Theorem 7.5, the presence of the WLP in characteristic zero can also be read off from the splitting type of the syzygy bundle. In fact, $I_{a, b, c, \alpha, \beta, \gamma}$ has the WLP if and only if its syzygy bundle has splitting type $(s+2, s+2, s+2)$ (see [20, Theorem 9.9]).

In [20], explicit formulae for the enumerations appearing in Theorem 7.5 are derived in various cases. However, even then determining the prime divisors of the enumerations can be challenging. In fact, this problem is open even in the special case of monomial complete intersections, though, recently, there has been progress in the case where the generators all have the same degree. Brenner and Kaid [12] gave an explicit description of when $R /\left\langle x^{d}, y^{d}, z^{d}\right\rangle$ has the WLP in terms of 
$d$ and the characteristic $p$. In particular, they proved a conjecture of [35] for the case $p=2$. This latter result is stated very concisely:

Theorem $7.7[\mathbf{1 2}]$. The algebra $k[x, y, z] /\left\langle x^{d}, y^{d}, z^{d}\right\rangle$ has the WLP in char $(k)=2$ if and only if $d=\left\lfloor\left(2^{n}+1\right) / 3\right\rfloor$ for some positive integer $n$.

The approach of [12] was via a theorem of Han computing the syzygy gap for an ideal of the form $\left\langle x^{d}, y^{d},(x+y)^{d}\right\rangle$ in $k[x, y]$. The analogous result in the case of more variables, that is, for $I=\left\langle x_{1}^{d}, \ldots, x_{n}^{d}\right\rangle(n \geq 4)$, has been obtained by Kustin and Vraciu in [34]. Independently, Cook made progress in deciding the Lefschetz properties of more general monomial complete intersections in positive characteristic (see [17]), addressing Question 7.1 (see also [36, Lemma 5.2], for a result in two variables).

In a different direction, Kustin, Rahmati and Vraciu [33] showed that $A=R /\left\langle x^{d}, y^{d}, z^{d}\right\rangle$ has the WLP in characteristic $p \neq 2$ if and only if its residue field has finite projective dimension as an $A$-module.

Acknowledgments. The first author is grateful to the organizers of the Midwest Commutative Algebra \& Geometry Conference for the invitation to speak, and both authors are grateful to Hal Schenck for his suggestion that they write this paper. The authors also thank David Cook II and the referee for helpful comments.

\section{ENDNOTES}

1. A simpler proof of this result has recently been given in [21].

Note added in proof. In the time since this paper was submitted, several important advances have been made in the study of the WLP which we have not been able to describe in this survey. Among these we single out $[\mathbf{7}, \mathbf{2 1}, \mathbf{2 2}, \mathbf{3 7}]$.

\section{REFERENCES}

1. M. Amasaki, The weak Lefschetz property for Artinian graded rings and basic sequences, preprint. 
2. D. Anick, Thin algebras of embedding dimension three, J. Algebra 100 (1986), 235-259.

3. D. Bernstein and A. Iarrobino, A nonunimodal graded Gorenstein Artin algebra in codimension five, Comm. Algebra 20 (1992), 2323-2336.

4. M. Boij, Graded Gorenstein Artin algebras whose Hilbert functions have a large number of valleys, Comm. Algebra 23 (1995), 97-103.

5. M. Boij and D. Laksov, Nonunimodality of graded Gorenstein Artin algebras, Proc. Amer. Math. Soc. 120 (1994), 1083-1092.

6. M. Boij, J. Migliore, R.M. Miró-Roig, U. Nagel and F. Zanello, The shape of a pure O-sequence, Mem. Amer. Math. Soc. 218 (2012).

7. - On the Weak Lefschetz Property for artinian Gorenstein algebras of codimension three, available at arXiv:1302.5742.

8. M. Boij and F. Zanello, Level algebras with bad properties, Proc. Amer. Math. Soc. 135 (2007), 2713-2722.

9. B. Boyle, The unimodality of pure O-sequences of type three in three variables, preprint.

10. - The unimodality of pure O-sequences of type two in four variables, preprint.

11. H. Brenner and A. Kaid, Syzygy bundles on $\mathbf{P}^{2}$ and the weak Lefschetz property, Illinois J. Math. 51 (2007), 1299-1308.

12. - A note on the weak Lefschetz property of monomial complete intersections in positive characteristic, Collect. Math. 62 (2011), 85-93.

13. K. Chandler, The geometric interpretation of Fröberg-Iarrobino conjectures on infinitesimal neighborhoods of points in projective space, J. Algebra 286 (2005), 421-455.

14. - Examples and counterexamples on the conjectured Hilbert function of multiple points, in: Algebra, geometry and their interactions, Contemp. Math. 448, American Mathematical Society, Providence, RI, 2007.

15. C. Chen, A. Guo, X. Jin and G. Liu, Trivariate monomial complete intersections and plane partitions, J. Commut. Algebra 3 (2011), 459-489.

16. CoCoATeam, CoCoA: A system for doing computations in commutative algebra, available at http://cocoa.dima.unige.it.

17. D. Cook II, The Lefschetz properties of monomial complete intersections in positive characteristic, J. Algebra 369 (2012), 42-58.

18. D. Cook II and U. Nagel, The weak Lefschetz property, monomial ideals, and lozenges, Illinois J. Math. 55 (2011).

19. - Hyperplane sections and the subtlety of the Lefschetz properties, J. Pure Appl. Algebra 216 (2012), 108-114.

20. — Enumerations deciding the weak Lefschetz property, preprint, available at arXiv:1105.6062.

21. - Enumerations of lozenge tilings, lattice paths, and perfect matchings and the weak Lefschetz property, available at arXiv:1305.1314.

22. R. Di Gennaro, G. Ilardi and J. Vallès, Singular hypersurfaces characterizing the Lefschetz properties, J. Lond. Math. Soc., to appear. 
23. L. Ein and R. Lazarsfeld, Syzygies and Koszul cohomology of smooth projective varieties of arbitrary dimension, Invent. Math. 111 (1993), 51-67.

24. J. Emsalem and A. Iarrobino, Inverse system of a symbolic power I, J. Algebra 174 (1995), 1080-1090.

25. D. Grayson and M. Stillman, Macaulay2, A software system for research in algebraic geometry, available at http://www.math.uiuc.edu/Macaulay2/.

26. B. Harbourne, H. Schenck and A. Seceleanu, Inverse systems, Gelfand-Tsetlin patterns and the weak Lefschetz property, J. Lond. Math. Soc. 84 (2011), 712-730.

27. T. Harima, T. Maeno, H. Morita, Y. Numata, A. Wachi and J. Watanabe, The Lefschetz properties, Lect. Notes Math. 2080, Springer-Verlag, New York, 2013.

28. T. Harima, J. Migliore, U. Nagel and J. Watanabe, The weak and strong Lefschetz properties for artinian K-algebras, J. Algebra 262 (2003), 99-126.

29. T. Hausel, Quaternionic geometry of matroids, Cent. Europ. J. Math. 3 (2005), 26-38.

30. J. Herzog and D. Popescu, The strong Lefschetz property and simple extensions, preprint, available on the arXiv at http://front .math.ucdavis.edu/0506.5537.

31. A. Iarrobino, Inverse system of a symbolic power III: Thin algebras and fat points, Compos. Math. 108 (1997), 319-356.

32. H. Ikeda, Results on Dilworth and Rees numbers of artinian local rings, Japan. J. Math. 22 (1996), 147-158.

33. A. Kustin, H. Rahmati and A. Vraciu, The resolution of the bracket powers of the maximal ideal in a diagonal hypersurface ring, J. Algebra 369 (2012), 256-321.

34. A. Kustin and A. Vraciu, The weak Lefschetz property for monomial complete intersections in positive characteristic, Trans. Amer. Math. Soc., to appear.

35. J. Li and F. Zanello, Monomial complete intersections, the weak Lefschetz property and plane partitions, Discrete Math. 310 (2010), 3558-3570.

36. M. Lindsey, A class of Hilbert series and the strong Lefschetz property, Proc. Amer. Math. Soc. 139 (2011), 79-92.

37. E. Mezzetti, R. Miró-Roig and G. Ottaviani, Laplace Equations and the Weak Lefschetz Property, Canad. J. Math. 65 (2013), 634-654.

38. J. Migliore and R. Miró-Roig, Ideals of general forms and the ubiquity of the weak Lefschetz property, J. Pure Appl. Algebra 182 (2003), 79-107.

39. J. Migliore, R. Miró-Roig and U. Nagel, Monomial ideals, almost complete intersections and the weak Lefschetz property, Trans. Amer. Math. Soc. 363 (2011), $229-257$.

40. - On the weak Lefschetz property for powers of linear forms, Algebra Number Theory 6 (2012), 487-526.

41. J. Migliore and U. Nagel, Reduced arithmetically Gorenstein schemes and simplicial polytopes with maximal Betti numbers, Adv. Math. 180 (2003), 1-63.

42. - Gorenstein algebras presented by quadrics, Collect. Math. 62 (2013), 211-233.

43. J. Migliore, U. Nagel and F. Zanello, A characterization of Gorenstein Hilbert functions in codimension four with small initial degree, Math. Res. Lett. 15 (2008), 331-349. 
44. J. Migliore and F. Zanello, The Hilbert functions which force the weak Lefschetz property, J. Pure Appl. Algebra 210 (2007), 465-471.

45. — The strength of the weak Lefschetz property, Illinois J. Math. 52 (2008), 1417-1433.

46. I. Novik and E. Swartz, Gorenstein rings through face rings of manifolds, Compos. Math. 145 (2009), 993-1000.

47. K. Pardue and B. Richert, Syzygies of semi-regular sequences, Illinois J. Math. 53 (2009), 349-364.

48. L. Reid, L. Roberts and M. Roitman, On complete intersections and their Hilbert functions, Canad. Math. Bull. 34 (1991), 525-535.

49. H. Schenck and A. Seceleanu, The weak Lefschetz property and powers of linear forms in $K[x, y, z]$, Proc. Amer. Math. Soc. 138 (2010), 2335-2339.

50. H. Sekiguchi, The upper bound of the Dilworth number and the Rees number of Noetherian local rings with a Hilbert function, Adv. Math. 124 (1996), 197-206.

51. S. Seo and H. Srinivasan, On unimodality of Hilbert functions of Gorenstein Artin algebras of embedding dimension four, Comm. Algebra 40 (2012), 2893-2905.

52. R. Stanley, Hilbert functions of graded algebras, Adv. Math. 28 (1978), 57-83.

53. R. Stanley, Weyl groups, the hard Lefschetz theorem, and the Sperner property, SIAM J. Algebr. Discr. Meth. 1 (1980), 168-184.

54. - The number of faces of a simplicial convex polytope, Adv. Math. 35 (1980), 236-238.

55. — Combinatorics and commutative algebra, 2nd ed., Progr. Math. 41, Birkhäuser, Boston, 1996.

56. J. Watanabe, The Dilworth number of Artinian rings and finite posets with rank function, in Commutative algebra and combinatorics, Adv. Stud. Pure Math. 11, Kinokuniya Co., North Holland, Amsterdam, 1987.

57. F. Zanello, A non-unimodal codimension 3 level h-vector, J. Algebra 305 (2006), 949-956.

58. F. Zanello and J. Zylinski, Forcing the strong Lefschetz and the maximal rank properties, J. Pure Appl. Algebra 213 (2009), 1026-1030.

Department of Mathematics, University of Notre Dame, Notre Dame, IN 46556

Email address: Juan.C.Migliore.1@nd.edu

Department of Mathematics, University of Kentucky, 715 Patterson Office Tower, Lexington, KY 40506

Email address: uwe.nagel@uky.edu 